\title{
Aprotinin and Nafamostat Mesilate in Liver Surgery: Effect on Blood Loss
}

\author{
Ilona T.A. Pereboom Marieke T. de Boer Robert J. Porte I. Quintus Molenaar \\ Department of Surgery, Section Hepatobiliary Surgery and Liver Transplantation, University Medical Center \\ Groningen, University of Groningen, Groningen, The Netherlands
}

\section{Key Words}

Aprotinin $\cdot$ Nafamostat mesilate $\cdot$ Liver transplantation $\cdot$ Hepatic resection

\begin{abstract}
The origin of blood loss during liver surgery is multifactorial. Surgical skill, technique, anesthesiological care, but also hyperfibrinolysis have been shown to play a role in the origin of bleeding during partial hepatectomy and liver transplantation. The latter has provided the scientific basis for the prophylactic use of antifibrinolytic drugs, such as aprotinin and nafamostat mesilate in liver surgery. Recently however, concern has been voiced about potential risks associated with aprotinin, including renal failure and thromboembolic events. In this review we discuss the efficacy and safety issues of aprotinin and nafamostat mesilate in liver surgery. We identified a total of 19 studies on the use of either aprotinin or nafamostat mesilate in liver surgery reported in the time period between 1966 and July 2006. The use of aprotinin or nafamostat mesilate in partial hepatectomies was studied in three studies. In 16 studies the use of aprotinin in liver transplantation was investigated. With respect to partial hepatectomy, improvements in surgical technique and anesthesiological care seem to be more important in reducing blood loss than the use of the antifibrinolytic drugs. Aprotinin may be indicated in a selected group of patients with cirrhosis undergoing liver resection, but further studies
\end{abstract}

\section{KARGER}

Fax +4161306 1234

E-Mail karger@karger.ch

www.karger.com (c) $2007 \mathrm{~S}$. Karger AG, Basel

$0253-4886 / 07 / 0244-0282 \$ 23.50 / 0$

Accessible online at:

www.karger.com/dsu in this specific group of patients will be needed. In liver transplantation, the use of aprotinin is associated with a significant reduction in blood loss and transfusion requirements of around $30-40 \%$. Results of prospective studies do not provide support for safety concerns as no increased risk for thromboembolic events or renal dysfunction has been observed in liver transplant patients treated with aprotinin. In conclusion, there is currently no scientific support for the routine use of aprotinin or nafamostat mesilate in patients undergoing partial hepatectomy, whereas the efficacy of aprotinin in liver transplantation is well established. More studies will be needed to address the safety aspects of aprotinin in patients undergoing liver surgery in more detail.

Copyright $\odot 2007$ S. Karger AG, Basel

\section{Introduction}

Surgeons have long recognized blood loss as the major impediment to the successful completion of an operation. It has been clearly shown during the last decades that reducing blood loss during major surgery improves outcome and reduces postoperative morbidity and mortality. In the past, surgery of the liver was a long and risky operation with large amounts of blood loss [1-3]. During the last decade, improvements in surgical techniques, surgical skills and anesthetic care have significantly reduced blood loss in liver surgery [4]. Despite these 
improvements, liver surgery still carries the risk of significant bleeding. Besides the obvious relation between perioperative blood loss and surgical skills, specific intraoperative hemostatic disorders, especially hyperfibrinolysis, have been shown to play a role [5-7].

Extensive research, especially in the field of liver transplantation, has improved the knowledge about specific intraoperative changes in coagulation that may occur during liver surgery. It has been shown that hyperfibrinolysis occurs due to increased levels of tissue-type plasminogen activator (t-PA), mainly during the anhepatic period of the operation [5-8]. t-PA leads to the conversion of plasminogen into plasmin hereby causing premature degradation of fibrin clots, resulting in increased bleeding. Investigations by Tsuzuki et al. [9] have shown that hyperfibrinolysis may also occur during partial hepatectomies, especially in cirrhotic patients. These observations have provided the scientific basis for the prophylactic use of antifibrinolytic drugs, such as serine protease inhibitors, in liver transplantation and during partial hepatectomies.

Aprotinin is a serine protease inhibitor, derived from bovine tissue. It has the ability to inhibit several proteases with serine at their active side, such as plasmin, kallikrein, trypsin and elastase. By blocking plasmin, serine protease inhibitors reduce hyperfibrinolysis and thus blood loss [5-7]. Apart from inhibiting plasmin, aprotinin may reversibly inhibit other proteases involved in the coagulation and inflammatory cascades. This may explain the ability of aprotinin to improve hemodynamic stability and to ameliorate the inflammatory response during major surgery $[10,11]$.

Another synthetic serine protease inhibitor with a similar mode of action is nafamostat mesilate (6-amino2 -naphthyl $p$-guanidinobenzoate dimethane-sulfonate). This drug is mostly used in Japan for the treatment of disseminated intravascular coagulation and acute pancreatitis, as well as an anticoagulant during hemodialysis in patients with a bleeding tendency [12-15]. Nafamostat mesilate inhibits various serine proteases generated during the coagulation cascade, as well as during inflammatory processes [16-18]. Nafamostat mesilate also inhibits coagulation factors such as factor VIIa [16] and thrombin [17], and has been found effective in treating patients with severe coagulopathy [14]. In addition, it may reduce fibrinolysis by blocking plasmin.

Aprotinin is widely used in liver transplantation [19]. The use of aprotinin and nafamostat mesilate during partial hepatectomies, however, is less extensively studied. Recently, two large observational studies in patients un- dergoing cardiac surgery have questioned the safety aspects of aprotinin, especially with respect to the risk of inducing renal dysfunction [20,21]. In addition, several case reports have suggested that aprotinin is associated with the risk of thromboembolic complications during liver transplantation [19]. In this review we will discuss the available literature and evaluate the efficacy and safety issues of both aprotinin and nafamostat mesilate in patients undergoing liver surgery.

\section{Methods}

Search Strategy and Selection of Trials

A systemic literature search was conducted. Literature was searched for the time period between 1966 and July 2006 using PubMed and Cochrane Library. The strategy was set up using the following single text words and combinations: aprotinin, liver transplantation, liver resection, hepatic resection, hepatectomy and nafamostat mesilate. Reference lists of relevant articles were cross-checked for other potentially relevant articles.

\section{Inclusion Criteria}

In the current review all reports of descriptive, randomized and non-randomized controlled studies on aprotinin or nafamostat mesilate in patients undergoing either partial hepatectomy or liver transplantation were included. The only exclusion criterion was non-English language publications.

\section{Results}

The trial selection process yielded a total of $156 \mathrm{ab}-$ stracts from the combined searches. Thirty-five appeared to be duplicates yielding a total of 121 studies which were retrieved for more detailed information. After critical appraisal of these publications, 19 studies were identified that met the inclusion criteria of our review. Those 19 studies were divided in two groups. The first group consisted of studies investigating the use of aprotinin or nafamostat mesilate in partial hepatectomy [22-24]. In two studies the use of nafamostat mesilate $[22,23]$ and in only one study the use of aprotinin in patients undergoing partial hepatectomy was evaluated [24]. The second group consisted of studies investigating the use of aprotinin in liver transplantation. In 13 studies, aprotinin and placebo or control groups were compared in patients undergoing liver transplantation $[8,25-38]$. In three studies a direct comparison of different dosages of aprotinin was made $[28,29,39]$. 


\section{Aprotinin and Nafamostat Mesilate in Partial Hepatectomies}

Although the use of aprotinin in liver transplantation has been studied extensively, only one randomized controlled trial on the use of aprotinin in partial hepatectomies has been conducted [24]. In this study the effect of aprotinin on blood loss and transfusion requirements was investigated in 97 patients undergoing partial hepatectomy in the period between 1992 and 1995. Patients were stratified according to diagnosis; cirrhotic versus normal livers and benign versus malignant tumors. Aprotinin was administered according to a large-dose scheme, which consisted of a loading dose of $2 \times 10^{6} \mathrm{kIU}$ of aprotinin over a 20-min period after induction of anesthesia, followed by a continuous infusion of $0.5 \times 10^{6} \mathrm{kIU} / \mathrm{h}$ administered by an infusion pump until skin closure. An additional bolus of $0.5 \times 10^{6} \mathrm{kIU}$ of aprotinin was infused after every 3 units of red blood cell (RBC) transfusion. Standardized anesthesiological and surgical techniques (Kelly fracture, pedicle occlusion) were used. 48 patients received aprotinin and 49 patients received placebo. Intraoperative blood loss, percentage of transfused patients, and total transfusion requirement per group were significantly lower in aprotinin-treated patients, compared with patients who received placebo $(1,217 \pm 966$ vs. $1,653 \pm$ $1,221 \mathrm{ml}, \mathrm{p}=0.048 ; 17$ vs. $39 \%, \mathrm{p}=0.02$, and 30 vs. 77 units of $\mathrm{RBC}, \mathrm{p}=0.015$, respectively). This study suggests that aprotinin reduces blood loss during partial liver resection; however, this study conducted in the mid-1990s has never been confirmed by another study.

The efficacy of nafamostat mesilate to reduce blood loss and transfusion requirements in patients undergoing a hepatic resection has been studied mostly in Japan [22, 23]. In one controlled trial the effect of nafamostat mesilate on coagulation and fibrinolysis was investigated in 22 patients with hepatocellular carcinoma, who underwent a partial hepatectomy [22]. Patients were divided into two groups: the first group (group 1) was a control group $(\mathrm{n}=11)$, the second group (group 2) received intra- and postoperative infusion of nafamostat mesilate $(\mathrm{n}=11)$. Nafamostat mesilate was continuously administered from the start of the operation until the end of the first postoperative day $(0.4 \mathrm{mg} / \mathrm{kg} / \mathrm{h})$. After postoperative day 1 the dose was reduced to $0.2 \mathrm{mg} / \mathrm{kg} / \mathrm{h}$ until postoperative day 3. In this study, nafamostat mesilate was shown to suppress coagulation activity, as reflected by a reduction in plasma levels of thrombin-antithrombin III complex and fibrinopeptide, and both during and immediately after the operation. Moreover, nafamostat mesilate signifi- cantly reduced fibrinolysis as reflected by a decreased euglobulin lysis activity both during and after the operation. However, no significant differences in blood loss during liver resection were seen between the two groups (blood loss in group 1: 1,393 $\pm 601 \mathrm{ml}$, vs. 1,509 \pm 590 $\mathrm{ml}$ in group 2). The number of patients who required $\mathrm{RBC}$ transfusions was not significantly different for the two groups (5/11 in group 1 vs. 2/11 in group 2) [22].

In a second study, also performed in Japan, nafamostat mesilate was studied in 20 patients undergoing extensive liver resections [23]. Patients were randomly allocated into two groups of 10 patients each. In one group, patients received nafamostat mesilate $(2 \mathrm{mg} / \mathrm{kg} /$ day $)$ during and after the operation for 7 days. The control group received standard postoperative intensive care, but no nafamostat mesilate. In this study, the use of nafamostat mesilate was also associated with reduced activation of the coagulation system during surgery. Fibrinolytic activity was increased after the operation in both groups and no differences in fibrinolysis were seen between the two groups.

The two controlled studies on the efficacy of nafamostat mesilate in reducing blood loss in patients undergoing partial hepatectomies both indicate that this drug has no major impact on bleeding during this type of surgery.

\section{Aprotinin in Liver Transplantation}

The use of aprotinin in patients undergoing liver transplantation was first reported by Neuhaus et al. [25] in 1989. These investigators described significant reductions in blood loss and transfusion requirements of 35 and $50 \%$, respectively, in a small group of patients, compared to historical controls. Similar observations have subsequently been reported by other groups, however, again in comparison to historical control groups [40-42]. A reduction in blood loss due to time-dependent factors such as improved surgical and anesthetic care could, therefore, not be excluded.

The European Multicenter Study on the use of Aprotinin in Liver Transplantation (EMSALT) was the first large randomized, controlled trial that provided definite proof for the blood loss reducing effect of aprotinin in liver transplantation [8]. In the EMSALT study, 137 patients were randomized into three groups: high-dose aprotinin $(\mathrm{n}=46)$, regular-dose aprotinin $(\mathrm{n}=43)$, or placebo $(\mathrm{n}=$ 48). Patients in the high-dose group received a loading dose of aprotinin of $2 \times 10^{6} \mathrm{kIU}$ followed by a continuous infusion of $1 \times 10^{6} \mathrm{kIU} / \mathrm{h}$ and an additional bolus of $1 \times$ 
$10^{6} \mathrm{kIU}$ before graft reperfusion. In the regular-dose group, patients received the same loading dose, followed by $0.5 \times$ $10^{6} \mathrm{kIU} / \mathrm{h}$ without an extra bolus. Administration of aprotinin or placebo was discontinued at $2 \mathrm{~h}$ after graft reperfusion. The total amount of intraoperative blood product transfusion was $40 \%$ lower in the high-dose group and $31 \%$ lower in the regular-dose group than the median value in the placebo group [8]. Very similar results have been found in another prospective, randomized, double-blind study performed at the Mayo Clinic [38]. In this study, 63 patients were randomized to receive either placebo or a low dose of aprotinin. 33 patients were administered an aprotinin according to the following scheme: loading dose of $1 \times 10^{6} \mathrm{kIU}$, followed by a continuous infusion of $0.25 \times$ $10^{6} \mathrm{kIU} / \mathrm{h}$. In the control group, 30 patients were administered equivalent volumes of saline. The total amount of intraoperative RBC transfusion was $28 \%$ lower in the lowdose aprotinin group, compared to placebo-treated patients. Although both the EMSALT study and the study performed at the Mayo Clinic did not show any significant differences in thromboembolic complications, both studies were not large enough to make any definitive conclusions about safety aspects.

To study the safety aspects of aprotinin in liver transplant recipients, we recently performed a systemic review and meta-analysis of all studies on antifibrinolytic drugs in liver transplantation, including a total of 1,407 patients [19]. The meta-analysis included the results of six randomized controlled trials in some of which also other antifibrinolytic drugs than aprotinin were studied. This analysis confirmed previous individual trials indicating that aprotinin significantly reduces RBC and fresh-frozen plasma (FFP) transfusion requirements during liver transplantation $[8,32,34-36,38]$. The overall incidence of side effects, such as venous thromboembolic events, was $1.9 \%(4 / 208)$ in aprotinin-treated patients versus $1.8 \%(3 / 168)$ in patients who received a placebo (odds ratio $0.92,95 \%$ CI $0.28-3.00, p=0.89$ ). Furthermore, analysis of perioperative hepatic artery thrombosis also showed no significant difference between aprotinintreated patients and patients receiving placebo (incidence of $1.4 \%(3 / 208)$ and of $3.0 \%(5 / 168)$ respectively, odds ratio $0.68,95 \%$ CI $0.17-2.75, \mathrm{p}=0.59)$ [19].

\section{Discussion}

In this review we evaluated the available literature on the efficacy and safety of the serine protease inhibitors aprotinin and nafamostat mesilate in reducing blood loss during partial hepatectomies and liver transplantation. During liver surgery, coagulopathy may occur especially in patients with cirrhosis, and this can lead to increased blood loss, disseminated intravascular coagulation or postoperative hepatic failure [9]. It is noted that primary hyperfibrinolysis may occur during hepatic resections and therefore the use of antifibrinolytic agents has been proposed as a method to reduce blood loss during partial hepatectomies and liver transplantation [22].

In 1997, Lentschener et al. [24] were the first to suggest that aprotinin may reduce blood loss and transfusion requirements in patients undergoing partial hepatectomy. Unfortunately, this has remained the only clinical study in which the efficacy and safety of aprotinin has been studied in patients undergoing liver resections. Therefore, this study has not been confirmed by another independent study. In addition, the amount of intraoperative blood loss observed in this trial was higher than what would be expected nowadays. Currently, most high-volume centers report partial hepatectomies without any blood cell transfusion requirements in up to $45 \%$ of the elective cases [43]. Improvements in surgical techniques, surgical skills and anesthetic care have contributed to a marked decrease of the number of patients needing blood products from over $80 \%$ to less than $40 \%$ [43]. Therefore, it is questionable whether the results obtained by Lentschener et al. can still be extrapolated to current practice. In general, it can be concluded that improvements in surgical techniques and anesthetic care have been more important to reduce blood loss during partial hepatectomies in patients with normal livers than the use of aprotinin or nafamostat mesilate. One aspect that has not been studied in great detail is the use of antifibrinolytic drugs in patients with cirrhosis undergoing partial hepatectomy. Cirrhotic patients are more prone to develop primary hyperfibrinolysis especially under stressful circumstances such as surgery. The use of antifibrinolytic drugs may be beneficial in these patients when undergoing a partial liver resection. This subject will need further research. Based on the current literature, the routine prophylactic use of antifibrinolytic drugs such as aprotinin and nafamostat mesilate cannot be recommended in patients undergoing partial hepatectomy.

Much more evidence exists with respect to the use of antifibrinolytic drugs in liver transplantation. The efficacy and safety of antifibrinolytics in liver transplantation has recently been evaluated in a systematic review and meta-analysis [19]. Results of this systematic review and meta-analysis clearly show that aprotinin significantly reduces RBC and FFP transfusion requirements 
during liver transplantation. The blood reducing effect could also be observed in the patients receiving another antifibrinolytic drug, tranexamic acid [19]. Since the amount of blood loss and thus transfusion requirements is decreasing due to improvements in surgical and anesthesiological care, careful examination of the risk-benefit ratio it is becoming more relevant. During the last decade, several possible side effects have been associated with the use of aprotinin. With respect to the use of aprotinin in liver transplantation, these concerns have mainly focused on the occurrence of thromboembolic complications. Intraoperative pulmonary embolism and intracardiac thrombus formation have been described in several case reports of patients receiving aprotinin during liver transplantation $[44,45]$. However, there are also several reports of these types of thromboembolic complications in patients undergoing liver transplantation who did not receive aprotinin or another antifibrinolytic drug [46-50]. The exact incidence of thromboembolic complications in patients undergoing liver transplantation is unknown. It has been suggested that the risk of venous thromboembolic complications is underestimated and that pulmonary embolisms may occur in up to $1 \%$ of the patients undergoing liver transplantation [46]. In the prospective studies reported so far, no difference in incidence of thrombotic complication has been reported between patients who received aprotinin or placebo. In our meta-analysis, venous thromboembolic events were also not significantly more observed in the aprotinin-treated patients [19]. Another potential side effect of aprotinin that was recently highlighted in two studies in patients undergoing cardiac surgery is the risk of renal failure [20, 21]. Based on a large observational multicenter study involving 4,374 patients, Mangano et al. [20] have recently suggested that the use of aprotinin in patients undergoing primary or complex coronary-artery surgery is associated with a doubling of the risk of renal failure requiring dialysis. In another single-center study, Karkouti et al. [21] also reported a higher incidence of renal insufficiency in patients receiving aprotinin during cardiac surgery, compared to tranexamic acid, another type of antifibrinolytic drug. Unfortunately, in both studies, patients were not randomized and baseline characteristics and condition of patients receiving aprotinin or other antifibrinolytic drugs were not comparable. Although the authors of both papers have tried to overcome this source of bias by performing multivariate analyses using propensity score adjustment, this statistical method also has its limitations and does not correct for unknown confounding variables. In liver transplantation, renal function in relation to the use of aprotinin has been evaluated in only one study in a subgroup of patients enrolled in the European multicenter trial EMSALT [51].This study did not reveal significant differences in postoperative renal function in patients receiving aprotinin, compared to patients receiving placebo. However, this study consisted of a total of 93 patients, which may have been too small to detect small, but clinically relevant differences in the incidence of renal failure.

In conclusion, based on this review of the literature on the efficacy and safety of aprotinin and nafamostat mesilate in liver surgery, we currently cannot recommend the prophylactic use of these two drugs in patients undergoing partial hepatectomy. There might be a role for aprotinin in a selected group of patients with cirrhosis and undergoing hepatic resection, but further studies in this subgroup of patients are needed. In liver transplantation, there is adequate scientific support for the prophylactic use of aprotinin. More studies, however, are needed to establish the impact of aprotinin on renal function in these patients. In addition, more and larger studies will be needed to settle the potential benefits and risks of aprotinin in comparison to other antifibrinolytic agents in liver transplantation.

\section{References}

1 Cunningham JD, Fong Y, Shriver C, et al: One hundred consecutive hepatic resections. Blood loss, transfusion, and operative technique. Arch Surg 1994;129:1050-1056.

-2 Bismuth H, Castaing D, Ericzon BG, et al: Hepatic transplantation in Europe. First report of the European Liver Transplant Registry. Lancet 1987;ii:674-676.

3 Starlz TE, Iwatsuki S, Van Thiel DH, et al: Evolution of liver transplantation. Hepatology 1982;5:614-636.
4 De Boer MT, Molenaar IQ, Hendriks HGD, Slooff MJH, Porte RJ: Minimizing blood loss in liver transplantation: progress through research and evolution of techniques. Dig Surg 2005;22:265-275.

5 Porte RJ, Knot EAR, Bontempo FA: Hemostasis in liver transplantation. Gastroenterology 1989;97:488-501.

6 Porte RJ: Coagulation and fibrinolysis in orthotopic liver transplantation: current views and insights. Semin Thromb Hemost 1993; 19:191-196.
7 Porte RJ, Bontempo FA, Knot EAR, et al: Systemic effects of tissue-plasminogen-activator-associated fibrinolysis and the relation with thrombin generation in orthotopic liver transplantation. Transplantation 1989;47: 987-984.

8 Porte RJ, Molenaar IQ, Begliomini B, et al: Aprotinin for reducing blood loss and transfusion requirements in orthotopic liver transplantation: a multicentre, randomized, double-blind study. Lancet 2000;355:13031309. 
>9 Tsuzuki T, Toyame K, Nakayasu K, et al: Disseminated intravascular coagulation after hepatic resection. Surgery 1990;107:172176.

-10 Molenaar IQ, Begliomini B, Martinelli G, et al: Reduced need for vasopressors in patients receiving aprotinin during orthotopic liver transplantation. Anesthesiology 2001;94: 433-438.

-11 Porte RJ, Hendriks, HGD, Slooff MJH: Blood conservation in liver transplantation: the role of aprotinin. J Cardiovasc Vasc Anesth 2004; $18: 31$ S-37S.

12 Akizawa T, Sato M, Kitaoka T, et al: The usefulness of the multi-enzyme inhibitor, nafamostat mesilate, in high bleeding risk hemodialysis. Proc Eur Dial Transplant Assoc 1985;22:334-338

13 Ohtake Y, Hirasawa H, Sugai T, et al: Nafamostat mesilate as anticoagulant on continuous hemofiltration and continuous hemodiafiltration. Contrib Nephrol. Basel, Karger, 1991, vol 93, pp 215-217.

14 Takahashi H, Takizawa S, Takewaki W, et al: Nafamostat mesilate (FUT-175) in the treatment of patients with disseminated intravascular coagulation. Thromb Haemost 1989; 62:372.

-15 Yoshikawa T, Murakami M, Furukawa Y, et al: Effects of FUT-175, a new synthetic protease inhibitor on endotoxin-induced disseminated intravascular coagulation in rats. Haemostasis $1983 ; 13: 374-378$.

- 16 Uchiba M, Okajima H, Abe H, et al: Effect of nafamostat mesilate, a serine protease inhibitor on tissue factor-factor VIIa complex activity. Thromb Res 1994;74:155-161.

17 Paques EP, Romisch J: Comparative study on the in vitro effectiveness of antithrombotic agents. Thromb Res 1991;64:11-21.

- 18 Okajima K, Uchiba M, Murakami K: Nafamostat mesilate. Cardiovasc Drug Rev 1995; 13:51-65.

19 Molenaar IQ, Warnaar N, Groen H, et al: Efficacy and safety of antifibrinolytic drugs in liver transplantation: a systematic review and meta-analysis. Am J Transplant 2007; 185-194.

20 Mangano DT, Tudor, IC, Dietzel C, et al: The risk associated with aprotinin in cardiac surgery. N Engl J Med 2006;354:353-365.

-21 Karkouti K, Beattie WS, Dattilo KM, et al: A propensity score case-control comparison of aprotinin and tranexamic acid in hightransfusion-risk cardiac surgery. Transfusion 2006;46:319-320

-22 Shimada M, Matsumata T, Shirabe K, et al: Effect of nafamostat mesilate on coagulation and fibrinolysis in hepatic resection. J Am Coll Surg 1994;178:498-502.

-23 Inagaki H, Nonami T, Kurowaka T: Effects of nafamostat mesilate, a synthetic protease inhibitor, or immunity and coagulation after hepatic resection. Hepatogastroenterology 1999;46:3223-3228.
24 Lentschener C, Benhamou D, Mercier FJ, et al: Aprotinin reduces blood loss in patients undergoing elective liver resection. Anesth Analg 1997;84:875-881.

25 Neuhaus P, Bechstein WO, Lefebre B, et al: Effect of aprotinin on intraoperative bleeding and fibrinolysis in liver transplantation. Lancet 1989;ii:924-925.

26 Mallet SV, Cos D, Burroughs AK, et al: The intraoperative use of Trasylol (aprotinin) in liver transplantation. Transplant Int 1991;4 227-230.

27 Grosse H, Lobbes W, Frambach M, et al: The use of high-dose aprotinin in liver transplantation: the influence on fibrinolysis and blood loss. Thromb Res 1991;63:287-297.

28 Himmelreich G, Muser M, Neuhaus P, et al: Different aprotinin applications influencing hemostatic changes in or thotopic liver transplantation. Transplantation 1992;53:132136.

29 Ickx B, Pradier O, DeGroote F, et al: Effect of two different dosages of aprotonin on perioperative blood loss during liver transplantation. Semin Thromb Hemost 1993;19:300 301.

30 Smith O, Hazlehurst G, Brozovic B, et al: Impact of aprotinin on blood transfusion requirements in liver transplantation. Transfus Med 1993;3:97-102.

31 Suarez M, Sangro B, Herrero JI, et al: Effectiveness of aprotinin in orthotopic liver transplantation. Semin Thromb Hemost 1993;19:292-296.

32 Welte M, Groh J, Azad S, et al: Effect of aprotinin on coagulation parameters in liver transplantation. Semin Thromb Hemost 1993;19:297-299.

33 Patrassi GM, Viero M, Sartori MT, et al: Aprotinin efficacy on intraoperative bleeding and transfusion requirements in orthotopic liver transplantation. Transfusion 1994;34:507-511.

34 Milroy SJ, Cottam S, Tan KC, et al: Improved haemodynamic stability with administration of aprotinin during orthotopic liver transplantation. Transfusion 1994;75:747751.

35 Marcel RJ, Stegall WC, Suit CT, et al: Continuous small-dose aprotinin controls fibrinolysis during orthotopic liver transplantation. Anesth Analg 1996;82:1122-1125.

-36 Garcia-Huete L, Domenech P, Sabate A, et al: The prophylactic effect of aprotinin on intraoperative bleeding in liver transplantation: a randomized clinical study. Hepatology 1997; 26:1143-1148.

37 Llamas P, Cabrera R, Gomez-Arnau J, Fernandez MN: Hemostasis and blood requirements in orthotopic liver transplantation with and without high-dose aprotinin. Haematologica 1998;83:338-346.
38 Findlay JY, Rettke SR, Ereth MH, et al: Aprotinin reduces red blood cell transfusion in orthotopic liver transplantation: a prospective, randomized, double-blind study. Liver Transplant 2001;7:802-807.

39 Soilleux H, Gillon MC, Mirand A, et al: Comparative effects of small and large aprotinin doses on bleeding during orthotopic liver transplantation. Anesth Analg 1995;80 349-352.

40 Hunt BJ, Cottam S, Segal H, et al: Inhibition by aprotinin of tPA-mediated fibrinolysis during orthotopic liver transplantation. Lancet 1990;336:381.

-41 Kraut E, Frey EK, Werle E: Über die Inaktivierung des Kallikreins. Hoppe Seylers Z Psychol Chem 1930;192:1-21.

42 Fritz H, Wunderer G: Biochemistry and applications of aprotinin, the kallikrein inhibitor from bovine organs. Arzneimittelforschung 1983;33:479-494.

43 Kooby DA, Stockman JRN, Ben-Porat LMS et al: Influence of transfusions on perioperative and long-term outcome in patients following hepatic resection for colorectal metastases. Ann Surg 2003;237:860-870.

44 Baubilier E, Cherqui D, Dominique C, et al: A fatal thrombotic complication during liver transplantation after aprotinin administration. Transplantation 1994;57:1664-1666.

45 Fitzsimons MG, Peterfreund RA, Raines DE: Aprotinin administration and pulmonary thromboembolism during orthotopic liver transplantation: report of two cases. Anesth Analg 2001;92:1418-1421.

46 Molenaar IQ, Porte RJ: Aprotinin and thromboembolism in liver transplantation: Is there really a causal effect? Anesth Analg 2002;94: 1367-1368.

47 Gologorsky E, De Wolf AM, Scott V, et al: Intracardiac thrombus formation and pulmonary thromboembolism immediately after graft reperfusion in seven patients undergoing liver transplantation. Liver Transpl 2001;7:783-789.

48 Navalgund AA, Kang Y, Sarner JB, et al: Massive pulmonary thromboembolism during liver transplantation. Anesth Analg 1988;67:400-402.

49 Ellis JE, Lichtor JL, Feinstein SB, et al: Right heart dysfunction, pulmonary embolism, and paradoxical embolization during liver transplantation. A transesophageal two-dimensional echocardiographic study. Anesth Analg 1989;68:777-782.

50 Lerner AB, Sundar E, Mahmood F, et al: Four cases of cardiopulmonary thromboembolism during liver transplantation without the use of antifibrinolytic drugs. Anesth Analg 2005;101:1608-1612.

51 Molenaar IQ, Begliomini B, Grazi GL, et al: The effect of aprotinin on renal function in orthotopic liver transplantation. Transplantation 2001;71:247-252. 